\title{
Frogs and some other interacting random walks models
}

\author{
Serguei Yu. Popov ${ }^{\dagger}$ \\ Instituto de Matemática e Estatística, Universidade de São Paulo, Rua do Matão 1010, CEP 05508-090, São Paulo \\ SP, Brasil \\ popoveime.usp.br
}

\begin{abstract}
We review some recent results for a system of simple random walks on graphs, known as frog model. Also, we discuss several modifications of this model, and present a few open problems. A simple version of the frog model can be described as follows: There are active and sleeping particles living on some graph. Each active particle performs a simple random walk with discrete time and at each moment it may disappear with probability $1-p$. When an active particle hits a sleeping particle, the latter becomes active.
\end{abstract}

Keywords: simple random walk, critical probability, shape theorem, recurrence

\section{Introduction}

The purpose of this note is to describe recent results and to propose some problems for future research for the system of interacting random walk called frog model. The basic version of this model can be informally described in the following way. Initially there is a random number of particles at each site of a graph $\mathcal{G}$. A site of $\mathcal{G}$ is singled out and called its root. All particles are sleeping at time zero, except for those that might be placed at the root, which are active. At each instant of time, each active particle may die with probability $(1-p)$. Once an active particle survives, it jumps on one of its nearest neighbors, chosen with uniform probability, performing a discrete time simple symmetric random walk (SRW) on $\mathcal{G}$. Up to the time it dies, it activates all sleeping particles it hits along its way. From the moment they are activated on, every such particle starts to walk, performing exactly the same dynamics, independent of everything else.

In this paper we consider several modifications of this model always keeping the following two common features:

- every particle performs a simple random walk, independently of everything else (although the moment when it starts may be random);

- there is no influx of particles to the system (but the particles may die), which distinguishes the models we consider here from e.g. branching random walks.

\footnotetext{
${ }^{\dagger}$ The author is thankful to CNPq (302981/02-0) for partial support. 
The model described above is a modification of a model for information spreading first proposed by K. Ravishankar. The idea is that every active particle has some information and it shares that information with a sleeping particle at the time the former jumps on the latter. Particles that have the information move freely helping in the process of spreading the information. Combustion chemical reactions provide another motivation for studying this process, cf. [8]. The model that we deal with in this paper is (with $p=1$ ) a discrete-time version of that proposed by R. Durrett (1996, private communication to F. Machado), who also suggested the term "frog model". Our study of the frog model with $p<1$ originated from a question asked by I. Benjamini to F. Machado in 2000.

To the best of our knowledge, the first published result on this model (with $p=1, \mathcal{G}=\mathbb{Z}^{d}$ and oneparticle-per-site initial configuration) is due to Telcs and Wormald [9] where it was referred to as the "egg model". They proved that, starting from that initial configuration, the origin will be visited infinitely often a.s. See Section 4 for more results about the probability of visiting the origin infinitely often.

Now, we give a formal definition of the model described above.

Let $\eta$ be a random variable taking values in $\mathbb{N}=\{0,1,2, \ldots\}$ such that $\mathbf{P}[\eta \geq 1]>0$. Let $\{\eta(x) ; x \in$ $\mathcal{G}\},\left\{\left(S_{n}^{x}(i)\right)_{n \in \mathbb{N}} ; i \in\{1,2,3, \ldots\}, x \in \mathcal{G}\right\}$ and $\left\{\left(\Xi_{p}^{x}(i)\right) ; i \in\{1,2,3, \ldots\}, x \in \mathcal{G}\right\}$ be independent sets of i.i.d. random variables defined as follows. For each $x \in \mathcal{G}, \eta(x)$ is an independent copy of $\eta$, and gives the initial number of particles at site $x$. If $\eta(x) \geq 1$, then for each $1 \leq i \leq \eta(x),\left(S_{n}^{x}(i)\right)_{n \in \mathbb{N}}$ is a discrete time SRW on $\mathcal{G}$ starting from $x$ (it describes the trajectory of $i$-th particle departing from $x$ ), and $\Xi_{p}^{x}(i)$, which stands for the lifetime of $i$-th particle departing from $x$, is a random variable whose law is given by $\mathbf{P}\left[\boldsymbol{\Xi}_{p}^{x}(i)=k\right]=(1-p) p^{k-1}, k=1,2, \ldots$, where $p \in[0,1]$ is a fixed parameter. Thus, the $i$-th particle at site $x$ follows the SRW $\left(S_{n}^{x}(i)\right)_{n \in \mathbb{N}}$ and dies (disappears) $\Xi_{p}^{x}(i)$ units of time after being activated. For $x \neq y$ let

$$
t(x, y)=\min _{1 \leq i \leq \eta(x)} \min \left\{n<\Xi_{p}^{x}(i): S_{n}^{x}(i)=y\right\}
$$

(note that, if the SRW is transient on $\mathcal{G}$, then $t(x, y)=\infty$ with positive probability). The moment when all the particles in $x$ are awakened is defined as

$$
T(0, x)=\inf \left\{t\left(x_{0}, x_{1}\right)+\cdots+t\left(x_{m-1}, x_{m}\right)\right\},
$$

where the infimum is over all finite sequences $0=x_{0}, x_{1}, \ldots, x_{m-1}, x_{m}=x$. Observe that $T(0, x)=\infty$ means that the site $x$ is never visited by active particles.

It is important to note that at the moment the particle disappears, it is not able to activate other particles (as first we decide whether the particle survives, and only after that the particle that survived is allowed to jump). Notice that there is no interaction between active particles, which means that each active particle moves independently of everything else. We denote by $\operatorname{FM}(\mathcal{G}, p, \eta)$ the frog model on the graph $\mathcal{G}$ with survival parameter $p$ and initial configuration given by independent copies of $\eta$ in each site of $\mathcal{G}$.

Next, we review known results for frogs, and propose some open problems.

\section{Shape theorem}

In this section we consider only the case $p=1$, i.e., the particles never die.

Let $\xi_{n}$ be the set of sites which were visited by active particles up to time $n$, namely, $\xi_{n}=\left\{y \in \mathbb{Z}^{d}\right.$ : $T(0, y) \leq n\}$. Define also $\bar{\xi}_{n}=\left\{y+(-1 / 2,1 / 2]^{d}: y \in \xi_{n}\right\} \subset \mathbb{R}^{d}$. 
Theorem 2.1 ([3], Theorem 1.1). Consider the model $\operatorname{FM}\left(\mathbb{Z}^{d}, 1, \eta\right)$. For any dimension $d \geq 1$ there is a nonempty convex set $\mathcal{A}=\mathcal{A}(d, \eta) \subset \mathbb{R}^{d}$ such that for almost all initial configurations, conditioned on $\{\eta(0) \geq 1\}$, we have for any $0<\varepsilon<1$

$$
(1-\varepsilon) \mathcal{A} \subset \frac{\bar{\xi}_{n}}{n} \subset(1+\varepsilon) \mathcal{A}
$$

for all $n$ large enough a.s.

For the particular case $\eta \equiv 1$ this result was obtained in [1] (Theorem 1.1). Naturally, the main tool for proving the above shape result is the Subadditive Ergodic Theorem. When applying that theorem, the most difficult part (especially in the dimension $d \geq 2$ ) was to prove that the mean time until some active particle comes to a site $x_{0}$ is finite for all $x_{0}$.

Note that, although Theorem 2.1 establishes the existence of the asymptotic shape $\mathcal{A}$, it is difficult to identify this shape exactly. Of course, $\mathcal{A}$ is symmetric, the origin belongs to the interior of $\mathcal{A}$, and $\mathcal{A} \subset \mathcal{D}$, where $\mathcal{D}=\left\{x=\left(x^{(1)}, \ldots, x^{(d)}\right) \in \mathbb{R}^{d}:\left|x^{(1)}\right|+\cdots+\left|x^{(d)}\right| \leq 1\right\}$. Also, note that if the initial configuration is augmented (i.e. some new particles are added), then the asymptotic shape (when it exists) augments as well. We are going to show that if the initial configuration is heavy enough, then the limiting shape $\mathcal{A}$ may contain some pieces of the boundary of $\mathcal{D}$ (a "flat edge" result), or even coincide with $\mathcal{D}$ (a "full diamond" result).

To formulate the "flat edge" result, we need some additional notation. For $d \geq 2$ and $1 \leq i<j \leq d$ let $\Lambda_{i j}=\left\{x=\left(x^{(1)}, \ldots, x^{(d)}\right) \in \mathbb{R}^{d}: x^{(k)}=0\right.$ for $\left.k \neq i, j\right\}$, and for $0<\beta<1 / 2$ let

$$
\Theta_{i j}^{\beta}=\left\{x=\left(x^{(1)}, \ldots, x^{(d)}\right) \in \Lambda_{i j}:\left|x^{(i)}\right|+\left|x^{(j)}\right|=1, \min \left\{\left|x^{(i)}\right|,\left|x^{(j)}\right|\right\} \geq \beta\right\} .
$$

Define $\Theta^{\beta}$ to be the convex hull of $\left(\Theta_{i j}^{\beta}\right)_{1 \leq i<j \leq d}$. We are interested now in the asymptotic shape in the frog model when the initial configuration is such that any site $x \in \mathbb{Z}^{d}$ contains exactly $m$ particles.

Theorem 2.2 ([1], Theorem 1.2). Consider the model $\mathrm{FM}\left(\mathbb{Z}^{d}, 1, m\right)$. For each $d \geq 2$ there exists $m_{0}=$ $m_{0}(d)$ such that for all $m \geq m_{0}$ there exists $0<\beta<1 / 2$ such that $\Theta^{\beta} \subset \mathcal{A}(d, m)$.

It is not difficult to see that, by monotonicity, the above result is verified for $\operatorname{FM}\left(\mathbb{Z}^{d}, 1, \eta\right)$ with $\eta$ such that $\eta \geq m_{0}$ a.s.

Next, we formulate the "full diamond" result:

Theorem 2.3 ([3], Theorem 1.2). Consider the model $\operatorname{FM}\left(\mathbb{Z}^{d}, 1, \eta\right)$. Suppose that for some positive $\beta<d$ and for all $n$ large enough we have

$$
\mathbf{P}[\eta(x) \geq n] \geq \frac{1}{(\log n)^{\beta}} .
$$

Then, Theorem 2.1 is verified with $\mathcal{A}=\mathfrak{D}$.

Remark. In [8], a continuous-time analogue of $\operatorname{FM}\left(\mathbb{Z}^{d}, 1,1\right)$ was considered. They prove the shape theorem (using the method different from that of $[1,3]$ ), and the fact that the local particle distribution tends to the product of Poissons. Also, it is proven there that when the dimension is large enough, the asymptotic shape is not the Euclidean ball (the speed in the axial direction is greater than the speed in the diagonal direction). 


\section{Phase transition}

For the model $\operatorname{FM}(\mathcal{G}, p, \eta)$, let us consider the following definition.

Definition 3.1. A particular realization of the frog model survives if for every instant of time there is at least one active particle. Otherwise, we say that it dies out.

Now we observe that $\mathbf{P}[\operatorname{FM}(\mathcal{G}, p, \eta)$ survives $]$ is nondecreasing in $p$ and define

$$
p_{c}(\mathcal{G}, \eta):=\inf \{p: \mathbf{P}[\operatorname{FM}(\mathcal{G}, p, \eta) \text { survives }]>0\} .
$$

As usual, we say that $\operatorname{FM}(\mathcal{G}, p, \eta)$ exhibits phase transition if $0<p_{c}(\mathcal{G}, \eta)<1$.

Now we present two lower bounds on $p_{c}(\mathcal{G}, \eta)$ which are obtained by a direct comparison with a Galton-Watson branching process.

Proposition 3.1 ([2], Propositions 1.1 and 1.2). If $\mathbf{E} \eta<\infty$, then for arbitrary graph $\mathcal{G}$ it holds that $p_{c}(\mathcal{G}, \eta) \geq(\mathbf{E} \eta+1)^{-1}$. If one makes an additional assumption that $\mathcal{G}$ is a graph of maximum degree $k$, then

$$
p_{c}(\mathcal{G}, \eta) \geq \frac{k}{1+(k-1)(\mathbf{E} \eta+1)} .
$$

Apart from comparison with a Galton-Watson branching processes, the main idea for proving most of the results of Section 3 is to think of the frog model as of (dependent) oriented percolation model. Namely, for each $x$, we consider its "virtual range", i.e., the set of sites visited by the particles originating from $x$, provided that they are eventually activated. To construct the oriented percolation process, we then draw oriented edges from $x$ to all sites belonging to the virtual range of $x$.

\subsection{Extinction and survival}

Next, we present some more general results concerning the extinction and the survival of the process, mainly for the cases of $\mathbb{Z}^{d}$ and regular trees.

We begin by showing that, under mild conditions on the initial number of particles, the process dies out a.s. in $\mathbb{Z}$ for every $p<1$. From now on, $a \vee b$ stands for $\max \{a, b\}$.

Theorem 3.1 ([2], Theorem 1.1). If $\mathbf{E} \log (\eta \vee 1)<\infty$, then $p_{c}(\mathbb{Z}, \eta)=1$.

Next, we find sufficient conditions to guarantee that the process becomes extinct for $p$ small enough in $\mathbb{Z}^{d}, d \geq 2$, and in $\mathbb{T}_{d}, d \geq 3$ (by $\mathbb{T}_{d}$ we mean a tree such that the degree of each vertex is $d$ ). Namely, for the case of tree, the result is given by

Theorem 3.2 ([2], Theorem 1.2). Suppose that there exists $\delta>0$ such that $\mathbf{E} \eta^{\delta}<\infty$. Then $p_{c}\left(\mathbb{T}_{d}, \eta\right)>0$, i.e., the process on $\mathbb{T}_{d}$ dies out a.s. for $p>0$ small enough.

For the case of $d$-dimensional lattice, the condition for the positivity of $p_{c}\left(\mathbb{Z}^{d}, \eta\right)$ is described by

Theorem 3.3 ([2], Theorem 1.3). Suppose that $\mathbf{E}(\log (\eta \vee 1))^{d}<\infty$. Then $p_{c}\left(\mathbb{Z}^{d}, \eta\right)>0$.

Now, let us state the results related to the survival of the process. The next theorem shows that for any nontrivial $\eta$ the frog model survives on $\mathbb{Z}^{d}, d \geq 2$, and on $\mathbb{T}_{d}, d \geq 3$, when the parameter $p$ is close enough to 1 .

Theorem 3.4 ([2], Theorems 1.4 and 1.5). If $\mathbf{P}[\eta \geq 1]>0$, then $p_{c}\left(\mathbb{Z}^{d}, \eta\right)<1$ for all $d \geq 2$ and $p_{c}\left(\mathbb{T}_{d}, \eta\right)<1$ for all $d \geq 3$. 
The method of proving Theorem 3.4 is based, of course, on the fact that the the usual percolation models on $\mathbb{Z}^{d}, d \geq 2$, and on $\mathbb{T}_{d}, d \geq 3$, percolate when the parameter is close enough to 1 .

The next result is the counterpart of Theorem 3.2. Note that Theorems 3.2 and 3.5 give the complete classification in $\eta$ of the frog model on $\mathbb{T}_{d}$ from the point of view of positivity of $p_{c}\left(\mathbb{T}_{d}, \eta\right)$.

Theorem 3.5 ([2], Theorem 1.6). If $\mathbf{E} \eta^{\delta}=\infty$ for every $\delta>0$, then $p_{c}\left(\mathbb{T}_{d}, \eta\right)=0$.

Besides, we are able to show that, for every fixed $d$, if $\eta$ has a sufficiently heavy tail, then $\operatorname{FM}\left(\mathbb{Z}^{d}, p, \eta\right)$ survives with positive probability for all values of $p \in(0,1)$ (which would be the counterpart of Theorem 3.3). However, we do not state this result now, since we will give a stronger result (cf. Theorem 4.3) later.

\subsection{Asymptotic values for the critical parameter and monotonicity}

The following theorem gives asymptotic values for critical parameters (compare with Proposition 3.1) for the case of $\mathbb{Z}^{d}$ and regular trees.

Theorem 3.6 ([2], Theorems 1.7 and 1.8). We have, for the case $\mathbf{E} \eta<\infty$,

$$
\lim _{d \rightarrow \infty} p_{c}\left(\mathbb{T}_{d}, \eta\right)=\lim _{d \rightarrow \infty} p_{c}\left(\mathbb{Z}^{d}, \eta\right)=\frac{1}{1+\mathbf{E} \eta}
$$

Remark. Observe that by truncating $\eta$ and using a simple coupling argument one gets that if $\mathbf{E} \eta=\infty$, then

$$
\lim _{d \rightarrow \infty} p_{c}\left(\mathbb{T}_{d}, \eta\right)=\lim _{d \rightarrow \infty} p_{c}\left(\mathbb{Z}^{d}, \eta\right)=0
$$

Note that Theorem 3.6 together with Proposition 3.1 suggest that there is some monotonicity of the critical probability in dimension. Then, a natural question to ask is the following:

Open Problem 3.1. Is it true that $p_{c}\left(\mathbb{Z}^{d}, \eta\right) \geq p_{c}\left(\mathbb{Z}^{d+1}, \eta\right)$ for all d (or at least for d large enough; also, can one substitute " $\geq$ " by " $>$ ")? What about the case of $\mathbb{T}_{d}$ ?

In fact, there is a more general question: for $\mathcal{G}_{1} \subset \mathcal{G}_{2}$, is it true that $p_{c}\left(\mathcal{G}_{1}, \eta\right) \geq p_{c}\left(\mathcal{G}_{2}, \eta\right)$, as happens for usual percolation models? In a recent paper [5] that last question was answered negatively. To formulate their result, let us call $\mathcal{G}^{*}(n)$ the graph whose set of vertices is $\cup_{x \in \mathbb{Z}^{2}} N_{n}(x)$ where $N_{n}(x)=$ $\{(x, 0),(x, 1), \ldots,(x, n)\}$. Moreover, its set of edges is such that for any $x \in \mathbb{Z}^{2}$ the vertex $(x, i)$ is neighbor of $(x, j)$ for $0 \leq i<j \leq n$. Besides, $(x, 0)$ is neighbor of $(y, 0)$ if $x, y$ are neighbors in $\mathbb{Z}^{2}$. In words, to construct the graph $\mathcal{G}^{*}(n)$, one have to attach complete graphs with $n$ vertices to each site of $\mathbb{Z}^{2}$.

Theorem 3.7 (see [5]). There exists $n_{0}$ such that for all $n \geq n_{0}$ we have $p_{c}\left(\mathbb{Z}^{2}, 1\right)<p_{c}\left(\mathcal{G}^{*}(n), 1\right)$.

\section{Recurrence and transience}

Again, consider the model $\operatorname{FM}(\mathcal{G}, p, \eta)$. One may also be interested in the study of other types of critical behaviour with respect to the parameter $p$. Consider the following

Definition 4.1. The model $\mathrm{FM}(\mathcal{G}, p, \eta)$ is called recurrent if

$\mathbf{P}[$ the root (the origin) is hit infinitely often in $\operatorname{FM}(\mathcal{G}, p, \eta)]>0$.

Otherwise, the model is called transient. 
Note that, even in the case when a single SRW on $\mathcal{G}$ is transient, it is still reasonable to expect that the frog model with $p=1$ is recurrent. For example, for the model $\operatorname{FM}\left(\mathbb{Z}^{d}, 1,1\right)$ the recurrence was established in [9]. However, establishing the recurrence property for that model is nontrivial; for example, consider the following

Open Problem 4.1. Is the model $\mathrm{FM}\left(\mathbb{T}_{d}, 1,1\right)$ recurrent?

Now, denote

$$
p_{u}(\mathcal{G}, \eta)=\inf \{p: \mathbf{P}[\text { the root is hit infinitely often in } \operatorname{FM}(\mathcal{G}, p, \eta)]>0\}
$$

(here, by definition, $\inf \emptyset=1)$; clearly, $p_{u}(\mathcal{G}, \eta) \geq p_{c}(\mathcal{G}, \eta)$ for every $\mathcal{G}$ and $\eta$. Now, we are interested in studying the existence of phase transition with respect to $p_{u}$.

First, we discuss some situations when the model is transient for every $p$, except possibly the case $p=1$.

Theorem 4.1 ([2], Theorems 1.9 and 1.10). Suppose that $\mathbf{E} \eta^{\varepsilon}<\infty$ for every $0<\varepsilon<1$. Then $p_{u}\left(\mathbb{T}_{d}, \eta\right)=$ 1. Now, suppose that $\mathbf{E}[\log (\eta \vee 1)]^{d}<\infty$. Then $p_{u}\left(\mathbb{Z}^{d}, \eta\right)=1$.

The next two theorems give sufficient conditions to have $p_{u}<1$ on trees and on $\mathbb{Z}^{d}$.

Theorem 4.2 ([2], Theorem 1.11). Suppose that there exists $\beta<\frac{\log (d-1)}{2 \log d}$ such that

$$
\mathbf{P}[\eta \geq n] \geq \frac{1}{n^{\beta}}
$$

for all $n$ large enough. Then $p_{u}\left(\mathbb{T}_{d}, \eta\right)<1$.

Theorem 4.3 ([2], Theorem 1.12). Suppose that there exist $\beta<d$ such that

$$
\mathbf{P}[\eta \geq n] \geq \frac{1}{(\log n)^{\beta}}
$$

for all $n$ large enough. Then $p_{u}\left(\mathbb{Z}^{d}, \eta\right)=0$.

(Compare this result with Theorem 2.3.)

Theorems 4.2 and 4.3 give sufficient conditions on the tail of the distribution of $\eta$ for the process to be recurrent when $p<1$. On the other hand, Theorem 4.1 shows that for the one-particle-per-site initial configuration the process is not recurrent even if the parameter $p<1$ is very close to 1 . The model with one-particle-per-site initial configuration being the most natural example one has to hand, a natural question is raised: What can be done (i.e., how can one modify the model) to make the model recurrent without augmenting the initial configuration? Notice that, by definition, in our model the lifetime of active particles is geometrically distributed. In order to find answers to that question, we change this and consider the situation when the lifetime has another distribution, possibly a more heavy-tailed one.

Let $\Xi$ be a nonnegative integer-valued random variable. Let us consider the frog model on $\mathcal{G}$ with oneparticle-per-site initial configuration, and the lifetimes of the particles after activation are i.i.d. random variables $\left(\Xi^{x}, x \in \mathcal{G}\right)$ having the same law as $\Xi$. This model is denoted by $\operatorname{FM}(\mathcal{G}, \Xi)$.

Theorem 4.4 ([2], Theorem 1.13). Suppose that one of the following alternatives holds:

- $\mathcal{G}=\mathbb{Z}$ and $\mathbf{E} \sqrt{\Xi}<\infty$, 
- $\mathcal{G}=\mathbb{Z}^{2}$ and $\mathbf{E} \frac{\Xi}{\log (\Xi \vee 2)}<\infty$,

- $\mathcal{G}=\mathbb{T}_{d}$ or $\mathbb{Z}^{d}, d \geq 3$ and $\mathbf{E} \Xi<\infty$.

Then $\operatorname{FM}(\mathcal{G}, \Xi)$ is transient.

Theorem 4.5 ([2], Theorem 1.14). For each dimension $d$ there exists $\beta_{d}>0$ such that if for all $n$ large enough one of the following alternatives holds

- $d=1$ and $\mathbf{P}\left[\Xi \geq n^{2}\right] \geq \beta_{1} n^{-1} \log \log n$,

- $d=2$ and $\mathbf{P}\left[\Xi \geq n^{2}\right] \geq \beta_{2} n^{-2}(\log n)^{2}$,

- $d \geq 3$ and $\mathbf{P}\left[\Xi \geq n^{2}\right] \geq \beta_{d} n^{-2} \log n$,

then $\operatorname{FM}\left(\mathbb{Z}^{d}, \Xi\right)$ is recurrent.

Theorems 4.1 and 4.4 are proved by making all the particles initially active, and getting an upper bound on the mean number of sites which send a particle to the origin. Theorems $4.2,4.3$, and 4.5 also are proved using a common approach: We think of $\mathcal{G}$ as a disjoint union of sets $\mathfrak{J}_{k}, k=1,2, \ldots$, of increasing sizes, such that with large probability (increasing with $k$ ), the set $\mathfrak{J}_{k}$ contains a lot of particles in the initial configuration. Besides, given that $\mathfrak{J}_{k}$ contains many particles, also with large probability (increasing with $k$ as well), the virtual paths of those particles will cover the whole set $\mathfrak{J}_{k+1}$ together with the origin, thus activating all particles placed originally in $\mathfrak{J}_{k+1}$. With a particular choice of that sequence of sets, the intersection of the events mentioned above occurs with strictly positive probability, which implies, consequently, that the process is recurrent (as in this case for each $k$ there is a particle from $\mathfrak{J}_{k}$ which visits the origin, and so the total number of particles visiting the origin is infinite).

As noted earlier in this paper, the question of recurrence of frog models is nontrivial even for the case of infinite lifetime (when, of course, the random walk of individual particle is transient). In view of that, the following problem is of interest. Given a collection of numbers $\left(0 \leq q(x) \leq 1, x \in \mathbb{Z}^{d} \backslash\{0\}\right), d \geq 3$, put a sleeping particle into site $x$ with probability $q(x)$. The configuration of sleeping particles is then fixed, and the process is started. The main question for such a model is: How can one distinguish transience from recurrence by looking at the initial density $q(x)$ ? The next theorem shows that the critical (i.e. separating transience from recurrence) rate of decay of the function $q(x)$ is $\|x\|^{-2}$ :

Theorem 4.6 ([7], Theorem 1.1). There exists $\alpha_{c r}=\alpha_{c r}(d), 0<\alpha_{c r}<\infty$, such that

(i) if $\alpha<\alpha_{c r}$ and $q(x) \leq \alpha\|x\|^{-2}$ for all x large enough, then the process is transient;

(ii) if $\alpha>\alpha_{c r}$ and $q(x) \geq \alpha\|x\|^{-2}$ for all $x$ large enough, then the process is recurrent.

The first part of Theorem 4.6, is proved by domination by branching random walk; the proof of the recurrence part is, however, more involved (we remark only that in the proof of the recurrence part there are some similarities with the proof of Theorem 4.5). 


\section{Frog model on finite graphs and other modifications}

\subsection{Finite graphs}

Here we describe some (current) results of the work [4].

In this section we suppose that $\mathcal{G}$ is a finite graph, and that the process starts with one-particle-persite initial configuration. In addition, we assume that active particles live some fixed time $t$, rather than having random geometrically distributed lifetime. Now, let $T_{\mathcal{G}}$ be the smallest value $t$ could be, in order to guarantee that with probability at least $1 / 2$ all vertices of $\mathcal{G}$ will be visited by active particles. The goal is now to estimate $T_{\mathcal{G}}$ for different types of graphs. In the sequel we state some of the (preliminary) results of [4].

Theorem 5.1. Let $\mathcal{G}$ be the the part of the one-dimensional lattice $\mathbb{Z}$ restricted to $\{0,1,2, \ldots, n\}$. Then $T_{\mathcal{G}}=O\left(\log ^{2} n\right)$.

Theorem 5.2. Let $\mathcal{G}$ be a complete graph with $n$ vertices. Then $T_{\mathcal{G}}=O(\log n)$.

The following result deals with the situation when it is much more difficult to visit all the vertices:

Theorem 5.3. Let $G$ be a complete graph with an extra vertex connected by an extra edge to each one of its vertices. We have in this situation that $T_{\mathcal{G}}=O\left(n \log ^{2} n\right)$.

Next, we present a result (which we hope to improve) for a two-dimensional box:

Theorem 5.4. Let $\mathcal{G}$ be the graph with the vertex set $\left\{x=\left(x_{1}, x_{2}\right) \in \mathbb{Z}^{2}: 1 \leq x_{1}, x_{2} \leq n\right\}$ and the edge set $\left\{[x, y]:\|x-y\|=1\right.$ and $\left.x, y \in \mathbb{Z}^{2}\right\}$. Then, $T_{\mathcal{G}}$ is such that

$$
C \log n \leq T_{\mathcal{G}} \leq \log ^{2} n .
$$

In order to approach the criticality in this model with $M=1$, we can perform the above construction with $\operatorname{FM}\left(\mathbb{Z}^{2}, p, 1\right)$ instead of $\operatorname{FM}\left(\mathbb{Z}^{2}, 1,1\right)$ (i.e., each active particle is killed with probability $(1-p)$ on each step, and, in addition, it is killed when it comes to a site where there is no sleeping particles, see Figure 2).

Open Problem 5.2. Prove that this process survives with positive probability for large enough $p$.

Simulations indicate that the critical value of $p$ is around 0.95 .

Now, we describe the second modification. The following model was proposed by Hervé Guiol (2000, private communication). Consider the frog model in $\mathbb{Z}^{d}$, with one-particle-per-site initial configuration, but with one important modification of the dynamics of the process: instead of dying, active particles become sleeping again with probability $(1-p)$, on each step (perhaps it is even better to consider this model with continuous time, to avoid defining voluntarily what happens in situations when e.g. a particle goes asleep in some site, while at the same moment another active particle comes to that site). Then, of course, if the original frog model survives for some $p$, then so does this modified model, but the situation with extinction is not at all clear:

Open Problem 5.3. Prove that this modified frog model becomes extinct for small enough $p$.

As we have seen above, the frog model with one-particle-per-site initial configuration always dies out in dimension 1 for any $p<1$, but here the situation is again not clear:

Open Problem 5.4. Does the modified frog model survive in dimension 1 with p close enough to 1 ? 


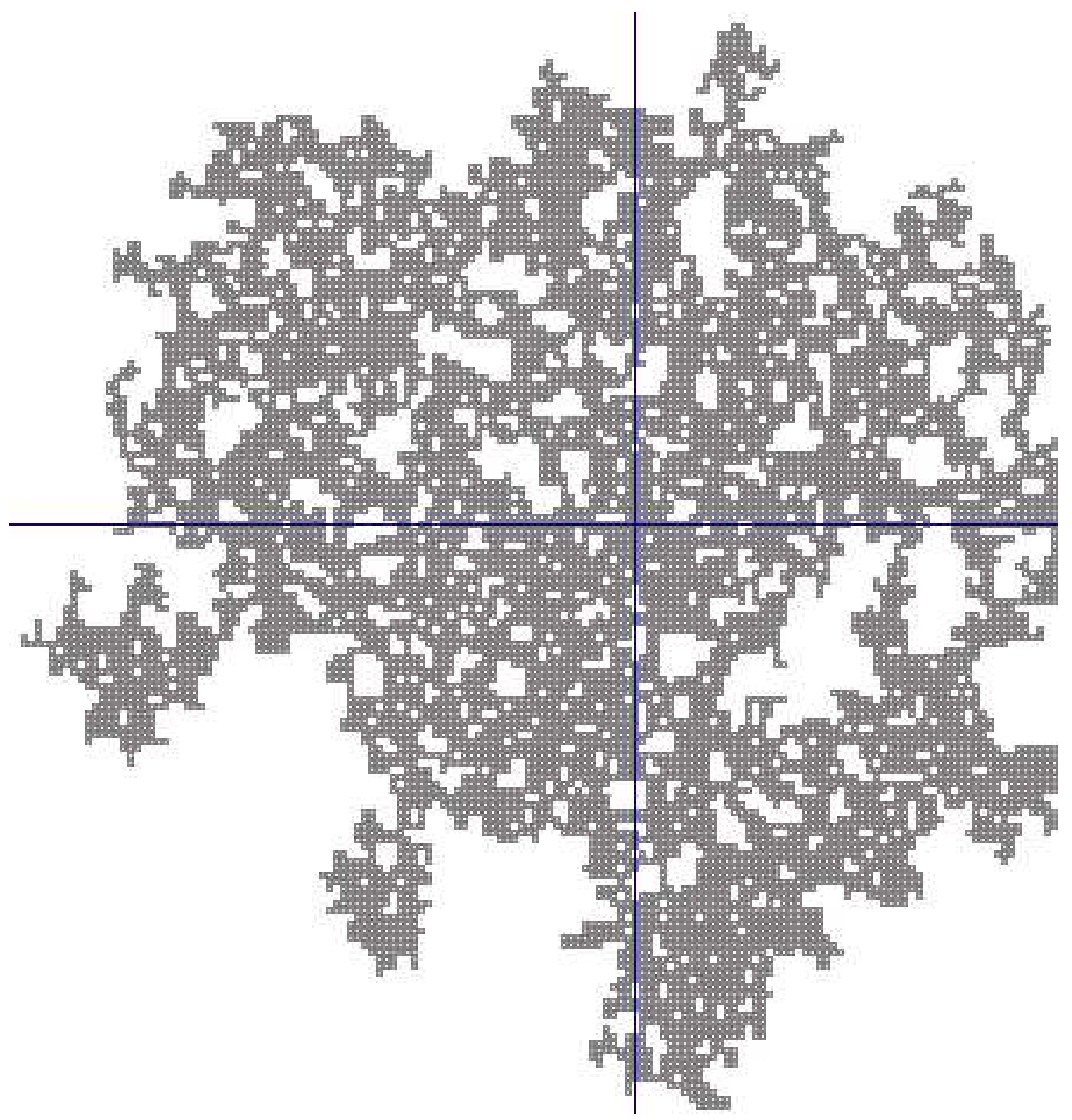

Fig. 1: The modified frog model with $M=1$ (and $p=1$ ), after 150 steps. Note that the holes inside the shape will never be visited. 


\subsection{Some other modifications}

In this section we describe two natural modifications of $\operatorname{FM}\left(\mathbb{Z}^{d}, p, 1\right)$, which give rise to some challenging open problems.

Before formulating the first modification, let us recall the shape result from [1] for $\mathrm{FM}\left(\mathbb{Z}^{d}, 1,1\right)$ (cf. Section 2). As we noted there, it is generally not possible to identify the asymptotic shape, so simulation seems to be the only way to get an idea about it. Performing the simulation for this model is, however, not an easy task, because, due to the linear growth speed, at time $t$ there are roughly $t^{d}$ moving particles in the system. To simplify the computations, one may note that any particle only encounters a finite number of sleeping particles during its lifetime (because an individual random walk has diffusive behavior, while the set of visited sites for the frog model grows linearly). So, if during the simulation an active particle does not awake anybody for a long time, it is reasonable to suppose that it will not reach the border of the growing shape anymore, and so we may remove it from the system without affecting the shape. Thus, let us introduce an integer parameter $M \geq 1$, and kill any active particle which did not wake up anybody for $M$ consecutive times. This idea was recently implemented (in dimension 2) by Fábio Machado and Lucas Meyer, and they found that indeed it permitted to speed up the computations. Then, just for curiosity, they put $M=1$, and the result was quite strange and interesting (see Figure 1): the process survived with high probability (at least 0.9 ), but, apparently, there was no linear growth, and the set of visited sites was fractal-like. Now, it is easy to prove that for $M$ large enough the process survives with positive probability, but the following problem remains open:

Open Problem 5.1. Prove that the process with $M=1$ survives with positive probability.

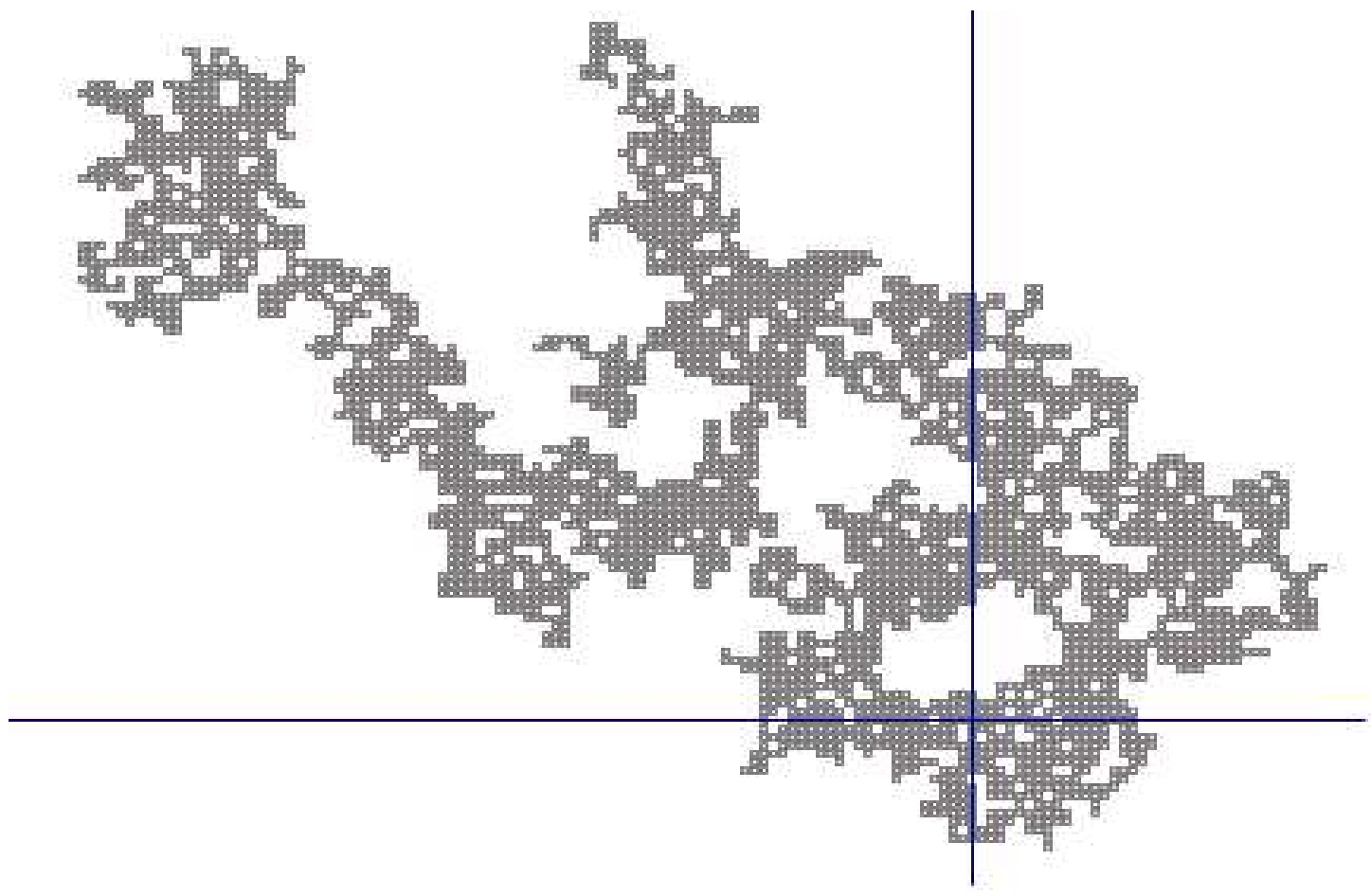

Fig. 2: The modified frog model with $M=1$ and $p=0.96$, after 300 steps. There are active particles, but only in the NW end of the shape. 


\section{Other models with interacting random walks}

In this section we consider the models with $\mathcal{G}=\mathbb{Z}^{d}$, with infinite lifetime, and where all the particles are active from the beginning.

\subsection{Shape theorem}

Let us start from some constant-density initial configuration of particles (it may be e.g. one-particle-persite, or a random configuration governed by Poisson product measure), and let those particles perform discrete-time SRWs independently. Moreover, suppose that the particles that are initially in 0 are infected. Infected particles transmit the disease to all the particles they meet, and there is no recovering. Similarly to Section 2, denote by $\xi_{n}$ the set of sites visited by infected particles up to the moment $n$. The following problem seems to be challenging:

Open Problem 6.1. Prove the analogue of Theorem 2.1 for this model.

Let us say a few words about the difficulties that arise when one tries to solve the above problem. A natural approach would be to consider the time $T(0, n x)$ when the infection comes to the site $n x$, and then, by using the subadditive ergodic theorem, to prove that $T(0, n x) / n$ converges to some limit as $n \rightarrow \infty$. In $[1,3]$, that was done in the following way: first, a set of auxiliary equally distributed random variables $T(k x,(k+1) x), k=0,1, \ldots$ was constructed in such a way that that sequence is mixing and the subadditive inequality

$$
T(0, n x) \leq T(0, x)+T(x, 2 x)+\cdots+T((n-1) x, n x)
$$

is verified for all the realizations of the process. Then, for the random variable $T(0, x)$ it was verified that its expectation is finite (that was the difficult part), which permitted us to use the subadditive ergodic theorem. Now, for this infection spreading model, it seems to be possible to prove, by using the method of $[1,3]$, that the mean time until the infection comes to $x$ is finite (with a little more work that would imply that $\xi_{n}$ grows linearly). What is difficult, is to construct a sequence of random variables as above, to which some subadditive ergodic theorem is applicable. To clarify a bit more this point, note that, even if we start from Poisson product measure (which is invariant with respect to this independent SRWs dynamics), the measure at the (random) moment when the infections gets to $x$ for the first time, is not the Poisson product measure.

\subsection{A competition model}

Here, exceptionally, we consider simple random walks with continuous time, and the dynamics is described as follows: there are red and blue particles; when a red particle comes to a site that contains blue particles, all those particles become red, and when a blue particle comes to a site that contains red particles, all those become blue. Initially, there is one red particle in the origin of $\mathbb{Z}^{d}, d \geq 3$, and one blue particle in each site of an infinite set $S \subset \mathbb{Z}^{d}$. The following result was proved in [6]:

Theorem 6.1. Let $\tau$ be the random moment when the last red particle becomes blue. We have that $\tau<\infty$ a.s. iff $\sum_{x \in S}\|x\|^{-(d-2)}=\infty$.

\section{References}

[1] O.S.M. Alves, F.P. Machado, S.Yu. Popov (2002) The shape theorem for the frog model. Ann. Appl. Probab. 12 (2), 533-546. 
[2] O.S.M. Alves, F.P. Machado, S.Yu. Popov (2002) Phase transition for the frog model. Electron. J. Probab. 7, paper no. 16, pp. 1-21.

[3] O.S.M. Alves, F.P. Machado, S.Yu. Popov, K. Ravishankar (2001) The shape theorem for the frog model with random initial configuration. Markov Process. Relat. Fields 7 (4), 525-539.

[4] I. Benjamini, L.R.G. Fontes, F.P. Machado, S.Yu. Popov. On an epidemic model on finite graphs. Work in progress.

[5] L.R.G. Fontes, F.P. Machado, A. Sarkar (2003) $p_{c}(\mathcal{G})$ for the frog model is not a monotonic function of $\mathcal{G}$. To appear in: J. Appl. Probab.

[6] I. Kourkova, S.Yu. Popov, M. Vachkovskaia (2003) A competition model with independent random walks. Preprint.

[7] S.Yu. Popov (2001) Frogs in random environment. J. Statist. Phys. 102 (1/2), 191-201.

[8] A.F. Ramírez, V. Sidoravicius (2002) Asymptotic behavior of a growth process of boundary branching random walks. C. R. Math. Acad. Sci. Paris 335 (10), 821-826.

[9] A. Telcs, N.C. Wormald (1999) Branching and tree indexed random walks on fractals. J. Appl. Probab. 36 (4), 999-1011. 